\title{
RESPUESTAS FISIOLÓGICAS Y NUTRIMENTALES DE VARIEDADES DE ARROZ A LA CONCENTRACIÓN DE ALUMINIO
}

\section{PHYSIOLOGICAL AND NUTRIMENTAL RESPONSES OF RICE VARIETIES TO ALUMINIUM CONCENTRATION}

\author{
Tania Marín-Garza ${ }^{1}$, Fernando C. Gómez-Merino ${ }^{2 *}$, Libia I. Trejo-Téllez ${ }^{1}$, Abel Muñoz-Orozco ${ }^{1}$, \\ Leticia Tavitas-Fuentes ${ }^{3}$, Leonardo Hernández-Aragón ${ }^{3}$ y Amalio Santacruz-Varela ${ }^{1}$
}

\begin{abstract}
${ }^{1}$ Colegio de Postgraduados, Campus Montecillo. Km 36.5 Carretera México-Texcoco. 56230, Montecillo, Texcoco, Estado de México. ${ }^{2}$ Colegio de Postgraduados, Campus Córdoba. Km 348 Carretera Córdoba-Veracruz. 94946, Amatlán de los Reyes, Veracruz, Tel.: 01 (55) 580459 11, Fax: 01 (55) 580459 12. ${ }^{3}$ Campo Agrícola Experimental Zacatepec, Instituto Nacional de Investigaciones Forestales, Agrícolas y Pecuarias. Km 0.5 Carretera Zacatepec-Galeana. 62780, Zacatepec, Morelos.

* Autor para correspondencia (fernandg@colpos.mx)
\end{abstract}

\section{RESUMEN}

En suelos ácidos $(\mathrm{pH}<5)$, la alta concentración de aluminio trivalente $\left(\mathrm{Al}^{3+}\right)$ en solución reduce la producción de los cultivos y resulta tóxico para la mayoría de las plantas, aunque hay respuestas diferenciales entre genotipos. En esta investigación se evaluó el efecto de tres concentraciones de $\mathrm{Al}(0,200$ y $400 \mu \mathrm{M})$ adicionadas a la solución nutritiva de Steiner al $20 \%$ en tres variedades mexicanas de arroz ('Temporalero', 'Huimanguillo', 'Tres Ríos') y una japonesa ('Koshihikari') cultivadas en invernadero, en un experimento factorial $4 \times 3$ con diseño completamente al azar. Ambos factores de estudio (variedad y concentración de Al) afectaron significativamente $(\mathrm{pH} \leq 5)$ la altura de planta al final del experimento. La var. 'Koshihikari' tratada con $400 \mu \mathrm{M}$ de $\mathrm{Al}$ mostró la menor altura ( $\mathrm{pH} \leq 5)$, en tanto que 'Temporalero' mostró la menor afectación de esta variable con el mismo tratamiento $(400$ $\mu M)$. Con excepción de 'Tres Ríos', las demás variedades presentaron daños a nivel tejido de raíz. Ambos factores y sus interacciones afectaron ( $\mathrm{P} \leq \mathbf{0 . 0 5})$ la concentración de $\mathrm{Al}, \mathrm{Ca}, \mathrm{K}, \mathrm{Mg}$ y $\mathrm{P}$ en hojas. Los niveles más bajos de estos nutrimentos se observaron en la variedad 'Koshihikari'. La variedad 'Tres Ríos' concentró menos Al que 'Temporalero' y 'Huimanguillo' (0.57 vs. 0.61 y $0.71 \mathrm{mg} \mathrm{kg}^{-1}$, respectivamente), y no disminuyó sus niveles de $\mathrm{Mg}$ con las aplicaciones de Al; además, 'Tres Ríos' mostró el menor efecto del Al en la concentración de $P$, pues aún con $400 \mu M$ de Al sólo disminuyó en $5 \%$ su contenido de $P$ respecto a las plantas testigo (0 $\mu \mathrm{M}$ Al). En cambio, 'Temporalero' disminuyó su contenido de $P$ en $38 \%$ respecto al testigo, y 'Huimanguillo' lo hizo en $55 \%$. Se concluye que la variedad mexicana de arroz 'Tres Ríos' es tolerante a Al porque no mostró daños en sus tejidos radicales y logró acumular menores niveles de $\mathrm{Al}$ y mayores niveles de $\mathrm{Mg}$ y $\mathrm{P}$ en hoja.

Palabras clave: Oryza sativa, tolerancia a aluminio, raíz, absorción de cationes.

\section{SUMMARY}

In acid soils $(\mathrm{pH}<5)$, the high concentration of trivalent aluminum $\left(\mathrm{Al}^{3+}\right)$ in solution reduces crop production and is toxic for most plant species, although different responses to $\mathrm{Al}$ are observed among genotypes. In this study we evaluated the effect of three $\mathrm{Al}$ concentrations $(0,200$ y $400 \mu \mathrm{M})$ added to the Steiner's nutrient solution at $20 \%$ in three Mexican rice varieties ('Temporalero', 'Huimanguillo', 'Tres Ríos') and a japanese one ('Koshihikari'); under greenhouse conditions, in a factorial $4 \times 3$ arrangement under a completely randomized experimental design. Both factors (variety and Al concentration) significatively $(\mathrm{pH} \leq 5)$ affected plant height at the end of the experiment. The 'Koshihikari' plants treated with $\mathbf{4 0 0}$ $\mu \mathrm{M}$ Al showed the shortest $(\mathrm{pH} \leq 5)$ plants, while 'Temporalero' plants were the less affected. With the exception of 'Tres Ríos', the other three varieties showed drastic damages on root cell structure. Both factors affected $(\mathrm{P} \leq \mathbf{0 . 0 5})$ the leaf concentrations of $\mathrm{Al}, \mathrm{Ca}, \mathrm{K}$, $\mathrm{Mg}$ and $\mathrm{P}$. The lowest levels of these nutrients were found in 'Koshihikari' plants. The Mexican variety 'Tres Ríos' concentrated lower amounts of $\mathrm{Al}$ in leaves compared to 'Temporalero' and 'Huimanguillo' (0.57 vs. 0.61 y $0.71 \mathrm{mg} \mathrm{kg}^{-1}$, respectively), and its Mg concentration was unaffected by the Al treatments; furthermore, the levels of $P$ in 'Tres Rios' were less affected by Al since they were diminished only by $5 \%$ in plants treated with $400 \mu \mathrm{M} \mathrm{Al}$, compared to the control plants (without $\mathrm{Al}$ in the solution), while the $\mathrm{P}$ levels decreased by $38 \%$ in 'Temporalero', and by $55 \%$ in 'Huimanguillo'. We conclude that the Mexican rice variety 'Tres Ríos' is tolerant to $\mathrm{Al}$ because it did not show root damages and accumulated less $\mathrm{Al}$ and more $\mathrm{Mg}$ y $\mathrm{P}$ in leaves.

Index words: Oryza sativa, aluminium tolerance, root, cations uptake.

\section{INTRODUCCIÓN}

Los suelos ácidos constituyen $30 \%$ de la superficie de la Tierra y casi $60 \%$ de los terrenos laborables. En México hay más de 14 millones de hectáreas de suelos ácidos distribuidos principalmente en los Estados de Veracruz, Tabasco, Oaxaca, Chiapas y Campeche (EcheverríaRubio, 2003). El factor más limitante de los suelos ácidos es la toxicidad del aluminio intercambiable $\left(\mathrm{Al}^{3+}\right)$, sobre todo cuando el $\mathrm{pH}$ del suelo es menor a 5.0. Dicha 
toxicidad se refleja en el crecimiento reducido del follaje y especialmente de las raíces, porque afecta la división celular de los tejidos radicales, la respiración y al mismo tiempo interfiere en la absorción, uso y transporte de nutrimentos (Poschenrieder et al., 2008).

En presencia de $\mathrm{Al}$ las raíces son gruesas pero quebradizas, con ápices y raíces laterales engrosados, que pueden llegar a tornarse de color café (Matsumoto, 2000), lo que las hace ineficientes en la absorción de nutrimentos y de agua. La concentración de $\mathrm{Al}$ en la raíz depende de la sensibilidad de las plantas a este metal, y la disminución del crecimiento se relaciona con el contenido de éste en dicho órgano. Muchas plantas poseen mecanismos de exclusión del metal desde la raíz, por lo que se les considera más tolerantes (Yamamoto et al., 1994).

El Al afecta de manera general la nutrición de la planta. Una elevada cantidad de calcio (Ca) disponible evita la entrada de $\mathrm{Al}$ a la raíz, sin afectar el crecimiento radical (Matsumoto et al., 1996; Rengel, 1992; Ma et al., 2002). Según Liu y Luan (2001), el Al inhibe la absorción de K en células oclusivas de Arabidopsis a través de un bloqueo interno, inhibición que es proporcional a la concentración de Al aplicada; así, con $10 \mu \mathrm{M}$ de Al se inhibe aproximadamente $50 \%$ de la absorción de $\mathrm{K}$, mientras que con $50 \mu \mathrm{M}$ de $\mathrm{Al}$ se inhibe en $79 \%$. Sin embargo, también se ha observado que en cebada (Hordeum vulgare L.) se estimula la absorción de $\mathrm{K}$ en raíces tratadas con $200 \mu \mathrm{M}$ de Al (Malkanthi et al., 1995).

La tolerancia de las plantas a la toxicidad por Al varía entre especies y entre variedades, especialmente en maíz (Zea mays L.), trigo (Triticum aestivum L.), cebada y arroz (Oryza sativa L.) (Kochian et al., 2004). En México, el arroz es considerado el tercer cereal en importancia por su superficie sembrada, producción y consumo (SIAP, 2009). Aunque se cuenta con un banco de germoplasma de arroz (Tavitas-Fuentes y HernándezAragón, 2000), se han hecho pocos estudios que caractericen dichos materiales, en especial sus respuestas al estrés abiótico que ocasionan los suelos ácidos donde se cultivan.

Para entender los efectos del $\mathrm{Al}$ en el metabolismo y la fisiología de variedades de arroz es necesario investigar si existen respuestas diferenciales en crecimiento y organización de células a nivel raíz, analizar cómo se afecta la absorción nutrimental, e identificar genotipos con mayor resistencia a este metal. El objetivo de la presente investígación fue evaluar el efecto de tres concentraciones de $\mathrm{Al}^{3+}$ adicionadas a la solución nutritiva, sobre la fisiología y la nutrición de cuatro variedades de arroz que se cultivan en México.

\section{MATERIALES Y MÉTODOS}

\section{Condiciones experimentales}

La investigación se hizo en condiciones de invernadero tipo cenital de estructura metálica y plástico calibre 600 (transmitancia luminosa de $50 \%$ ) ubicado en Montecillo, Texcoco, Estado de México (a $19^{\circ} 20^{\prime} \mathrm{LN}, 9^{\circ} 53^{\prime} \mathrm{LO}$, $2250 \mathrm{msmn})$.

\section{Material vegetal}

El experimento inició con la germinación de las semillas de cuatro variedades de arroz (las mexicanas 'Temporalero', 'Huimanguillo', 'Tres Ríos' y la japonesa 'Koshihikari'), que se hizo en cajas petri sobre papel filtro humedecido con agua destilada. Ocho días después de la germinación, las plántulas fueron trasplantadas a charolas de plástico que contenían suelo esterilizado, donde permanecieron hasta alcanzar $35 \mathrm{~d}$ de edad y $8 \mathrm{~cm}$ de altura. Luego, las plantas fueron trasplantadas a macetas de $3 \mathrm{~L}$ de capacidad que contenían la solución nutritiva de Steiner (a $20 \%, \mathrm{pH}$ 5.5), y mantenidas ahí por una semana como periodo de adaptación.

\section{Tratamientos y diseño experimental}

Posterior a la semana de adaptación se aplicaron los tratamientos de $\mathrm{Al}$, en un experimento con arreglo factorial $4 \times 3$, donde el factor 1 fue la variedad de arroz ('Temporalero', 'Huimanguillo', 'Tres Ríos' y 'Koshihikari'), y el factor 2 fue la concentración de $\mathrm{Al}^{3+}$ $(0,200$ y $400 \mu \mathrm{M})$. Los 12 tratamientos fueron distribuidos en un diseño experimental completamente al azar, en el que una planta fue considerada como unidad experimental, con 12 plantas por tratamiento, distribuidas en tres macetas de $3 \mathrm{~L}$ de capacidad (cuatro plantas por maceta).

\section{Solución nutritiva}

Para el crecimiento de las plantas se empleó la solución nutritiva de Steiner a $20 \%$, la cual contenía los siguientes iones (en molc m${ }^{-3}$ ): 2.4 de $\mathrm{NO}_{3}^{-} ; 0.2 \mathrm{de}_{2} \mathrm{PO}_{4}^{-} ; 1.4$ de

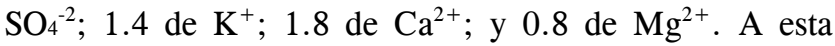
solución se le adicionó una mezcla de micronutrimentos en las siguientes concentraciones $\left(\mathrm{mg} \mathrm{L}^{-1}\right): 1.6 \mathrm{de} \mathrm{Mn}$; 0.11 de $\mathrm{Cu} ; 0.865$ de B; 0.023 de $\mathrm{Zn}$; y 5.0 de Fe, en la que $\mathrm{Mn}, \mathrm{Cu}$ y $\mathrm{Zn}$ fueron suministrados en forma de sulfatos; B como $\mathrm{H}_{3} \mathrm{BO}_{3}$; y Fe como quelato (Fe-EDTA), según lo descrito por Steiner y van Winden (1970). El aluminio fue abastecido a las soluciones nutritivas en forma de $\mathrm{AlCl}_{3} \cdot 6 \mathrm{H}_{2} \mathrm{O}$; el $\mathrm{pH}$ de la solución fue ajustado a un valor de 4.2, para garantizar que el $\mathrm{Al}$ permaneciera 
en su forma iónica $\mathrm{Al}^{3+}$. La solución nutritiva fue renovada cada $48 \mathrm{~h}$, con un ajuste de $\mathrm{pH}$ a 4.2 a las $24 \mathrm{~h}$. La solución nutritiva no fue oxigenada, porque el arroz es una especie que se cultiva bajo condiciones de anoxia.

\section{Variables medidas}

Altura de planta. En las semanas 2 y 4 del experimento se midió la altura de las plantas, y se tomaron fotografías para registrar el tamaño de la parte aérea y de raíces.

Registro fotográfico de daños en raíz. Para visualizar el daño que ocasiona el $\mathrm{Al}$ a nivel tejido, al final del experimento (semana 4) se tomaron dos raíces de dos plantas por tratamiento, en las que se hicieron cortes transversales con la técnica del corte fino. Los cortes de $2 \mathrm{~mm}$ de grosor, se fijaron con glutaraldehído $3 \%$ en amortiguador de fosfatos de Sorensen $0.1 \mathrm{M}$ y pH 7.2 durante $24 \mathrm{~h}$. Posteriormente se aplicaron tres lavados seriados con el amortiguador de fosfatos antes mencionado, de 1 min en cada cambio. Los cortes se deshidrataron en una serie de soluciones de etanol $(30,40,50,60,70$, $80,90,100$ y $100 \%$, v/v) por 45 min en cada una. Las muestras deshidratadas se pusieron en una secadora de punto crítico (marca Sandri-780A®), se fijaron sobre portamuestras con una cinta doble adhesiva de cobre y se recubrieron con oro durante 4 min en una ionizadora (Ion Sputter JFC-1100®, Jeol, Fine Coat). Las muestras se observaron en un microscopio electrónico de barrido marca JEOL ${ }^{\circledR}$ modelo JSM-6390 operado a $10 \mathrm{Kv}$.

Concentración de nutrimentos. Después de cuatro semanas de tratamiento con $\mathrm{Al}$, las plantas fueron cosechadas. Follaje y raíces fueron cortados y secados para la determinación de los contenidos de $\mathrm{Al}, \mathrm{Ca}, \mathrm{K}, \mathrm{Mg}$ y P. Las muestras fueron sometidas a digestión húmeda del material seco con una mezcla de ácidos perclórico y nítrico (Alcántar y Sandoval, 1999). Los extractos obtenidos fueron leídos en un equipo de espectrometría de emisión atómica de inducción por plasma (ICP-AES) marca VARIAN® modelo Liberty II.

Análisis estadístico. Los datos obtenidos del experimento fueron analizados mediante el paquete estadístico SAS (SAS Institute, 1990), con el procedimiento ANOVA; las medias observadas fueron comparadas por la prueba de Tukey ( $\alpha=0.05$ y 0.01$)$.

\section{RESULTADOS Y DISCUSIÓN}

\section{Altura de plantas}

El factor variedad afectó de manera significativa ( $\mathrm{P} \leq$ 0.01 ) a la altura, tanto en la semana 2 como en la 4
(Cuadro 1). El factor concentración de Al sólo afectó la altura en la semana $4(\mathrm{P} \leq 0.05)$. La interacción entre variedad $\mathrm{x}$ concentración de $\mathrm{Al}$ sólo fue significativa $(\mathrm{P} \leq$ $0.05)$ en la semana 2.

Cuadro 1. Efecto de variedad, concentración de Al y de su interacción, en la altura de planta en arroz.

\begin{tabular}{lccc}
\hline Fuente de variación & $\begin{array}{c}\text { Grados de } \\
\text { libertad }\end{array}$ & $\begin{array}{c}\text { Semana } \\
2\end{array}$ & $\begin{array}{c}\text { Semana } \\
4\end{array}$ \\
\hline Variedad & 3 & $* *$ & $* *$ \\
Concentración de Al & 2 & ns & $* *$ \\
Variedad x Concentración de Al & 6 & $*$ & ns \\
\hline ns = No significativo; $*$ Significativo a $5 \% ; * *$ Significativo a $1 \%$.
\end{tabular}

Después de cuatro semanas de tratamiento la menor altura $(12.3 \mathrm{~cm})$ fue observada en la variedad 'Koshihikari' tratada con $400 \mu \mathrm{M}$ de $\mathrm{Al}$ en la solución, en tanto que las tres variedades mexicanas resultaron menos afectadas pues alcanzaron una altura promedio de 16.2 $\mathrm{cm}$, sin diferencias significativas entre ellas (Figura 1).

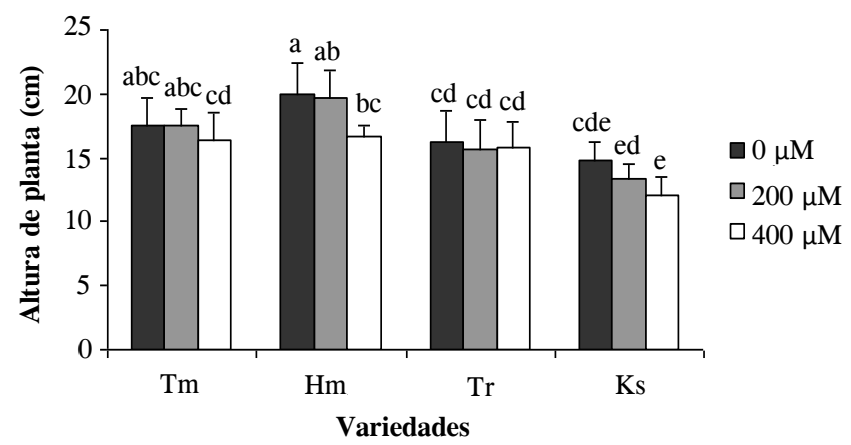

Figura 1. Altura de plata de cuatro variedades de arroz crecidas durante cuatro semanas en soluciones nutritivas con distintas concentraciones de Al. Variedades Tm, 'Temporalero'; Hm, 'Huimanguillo'; Tr, 'Tres Ríos'; y Ks, 'Koshihikari'. Letras sobre las barras indican diferencias significativas entre tratamientos (Tukey, 0.05). Líneas verticales sobre las barras indican desviaciones estándares.

La Figura 2 ilustra el tamaño que alcanzaron las plantas después de cuatro semanas de tratamiento con Al. En todas las variedades, el aumento en la concentración del metal en la solución provocó disminución del tamaño de planta. De las cuatro variedades, la que presentó menores efectos visibles de toxicidad de Al fue 'Tres Ríos'. A este respecto, Macedo y Jan (2008) observaron que en las variedades de arroz sensibles a $\mathrm{Al}$ el transporte de $\mathrm{Ca}, \mathrm{P}$ y Mn de la raíz hacia los brotes fue menor que en las resistentes, ya que éstas alcanzaron mayor altura que las primeras.

Se han reportado respuestas diferenciales a Al tanto entre especies y subespecies como entre variedades. Watanabe y Okada (2005) demostraron que las variedades de arroz más tolerantes a $\mathrm{Sr}$ y Ba también lo fueron para Al (japonica > indica). Jan (2005) comparó las 
respuestas de tres variedades de arroz ('BG35', 'DA141' y 'IR45') al aluminio (0, 140, 280 o $560 \mu \mathrm{M}$ de $\mathrm{Al})$ en la solución nutritiva, y encontró que el cultivar 'BG35' tolerante a Al mostró mayor crecimiento que la variedad sensible 'IR45'. En Lespedeza bicolor, Sun et al. (2008) encontraron mayor resistencia a $\mathrm{Al}$ que en $L$. cuneata y postularon que la estimulación de la resistencia a $\mathrm{Al}$ por aplicaciones de $\mathrm{P}$ en especies resistentes, puede estar asociada con una mayor eficiencia en la acumulación y removilización de $\mathrm{P}, \mathrm{y}$ con mayor exclusión de $\mathrm{Al}$ de los ápices de la raíz después de aplicar $\mathrm{P}$, más que con un incremento en la exudación de ácidos orgánicos de la raíz.

Macedo y Jan (2008) reportaron que las variedades resistentes 'IRAT112' y 'IR6023' muestran menor absorción y traslocación de Al de las raíces a los tallos, combinado con un transporte más eficiente de $\mathrm{Ca}, \mathrm{P}$ y $\mathrm{Mn}$. Al estudiar la respuesta a $\mathrm{Al}$ en 12 genotipos de Stylosanthes, Du et al. (2009) encontraron que hubo una amplia variación genotípica en crecimiento y absorción de $P$ en condiciones de acidez del suelo ( $y$, por ende, baja disponibilidad de $\mathrm{P}$ ); de los 12 genotipos evaluados, 'TPRC2001-1' mostró mayor tolerancia a Al y mayor eficiencia en el uso de $\mathrm{P}$ que el resto de genotipos.

\section{Efectos del $\mathbf{A l}$ en la estructura de la raíz}

En esta investigación se demostró que la aplicación de Al provoca destrucción en la raíz del arroz (Figura 3). En el tratamiento testigo $(0 \mu \mathrm{M}$ de $\mathrm{Al})$ los tejidos no presentaron destrucción, mientras que las plantas sometidas a estrés por Al mostraron desórdenes en la organización celular en raíces, y la magnitud de tal desorden correspondió al nivel de $\mathrm{Al}$ con el que fueron tratadas, excepto en la variedad 'Tres Ríos', en la que no hubo alteraciones en la organización celular, por lo que no se deduce que es una variedad que puede tolerar la toxicidad por Al. Hirano y Hijii (1998) demostraron los efectos de la toxicidad causada por Al en la morfología de la raíz, y Lenoble et al. (1996) reportaron que la aplicación de B en la solución nutritiva puede prevenir daños ocasionados por Al. La protección que confiere el $\mathrm{B}$ que señalaron otros autores, también puede ocurrir en este estudio ya que se usó B en la solución nutritiva, y se manifestó en todas las variables aquí analizadas: crecimiento de raíz, elongación celular en la raíz primaria. Resultados similares fueron observados por Blancaflor et al. (1998) en maíz. En especies y variedades resistentes se han observado mecanismos similares a los que confiere el B.

El aluminio interfiere en la división celular en ápices radicales y en raíces laterales porque incrementa la rigidez de la pared celular por vinculación de pectinas cruzadas, reduce la replicación del DNA mediante el aumento de la rigidez de la doble hélice, e interfiere con la actividad enzimática que regula la fosforilación de azúcares, la deposición de polisacáridos en la pared celular y la absorción, transporte y el uso de varios nutrientes esenciales como $\mathrm{Ca}, \mathrm{Mg}, \mathrm{K}, \mathrm{P}$ y $\mathrm{Fe}$ (Aimi et al., 2009; Du et al., 2009).

\section{Concentración de nutrimentos}

El análisis nutrimental demostró que la variedad, la concentración de $\mathrm{Al}$ y la interacción entre ambos factores ejercieron efectos significativos $(\mathrm{P} \leq 0.05)$ sobre la concentración de $\mathrm{Al}, \mathrm{Ca}, \mathrm{K}, \mathrm{Mg}$ y $\mathrm{P}$ en hojas de arroz (Cuadro 2). El efecto del factor variedad en la concentración de $\mathrm{Al}, \mathrm{Ca}, \mathrm{K}, \mathrm{Mg}$ y $\mathrm{P}$ se muestra en el Cuadro 3. En todos los casos la mayor concentración de estos cationes de observó en la variedad 'Temporalero'. Las variedades 'Huimanguillo', 'Tres Ríos' y 'Koshihikari' presentaron los menores valores de Ca y $\mathrm{Mg}$, en tanto que para $\mathrm{K}$ y $\mathrm{P}$ los valores más bajos se registraron en las variedades 'Huimanguillo' y 'Tres Ríos'.
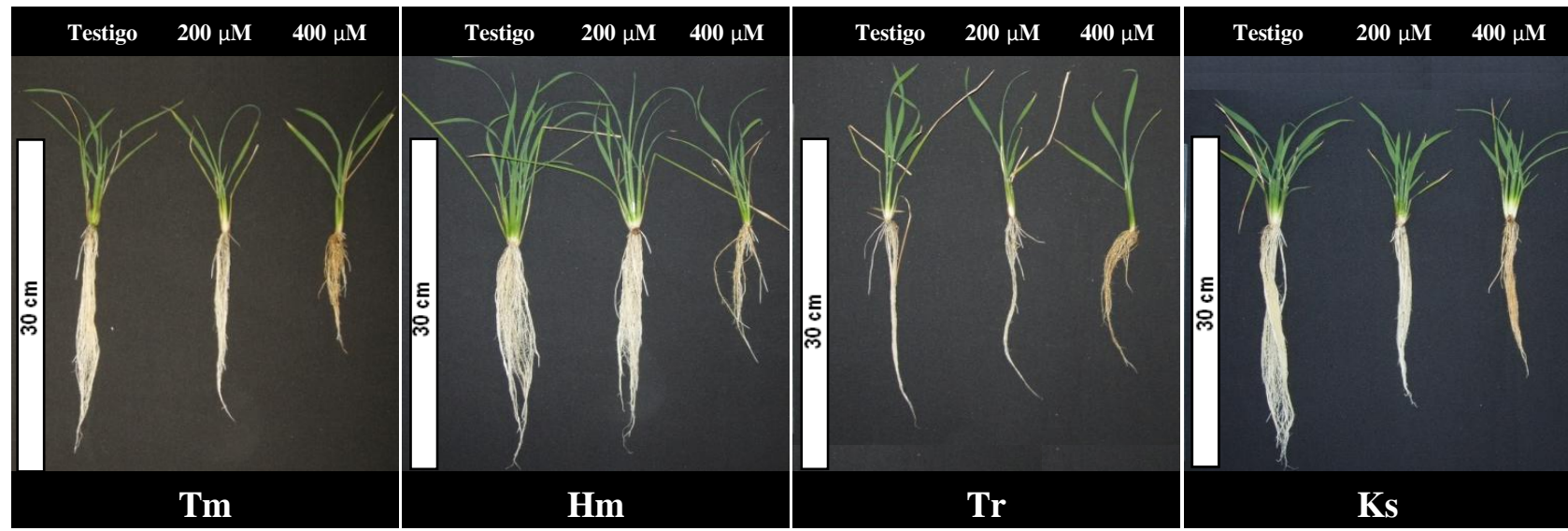

Figura 2. Tamaño de planta alcanzado por cuatro variedades de arroz crecidas durante cuatro semanas en soluciones nutritivas con distintas concentraciones de Al. Variedades: Tm, 'Temporalero'; Hm, 'Huimanguillo'; Tr, 'Tres Ríos'; y Ks, 'Koshihikari'. 


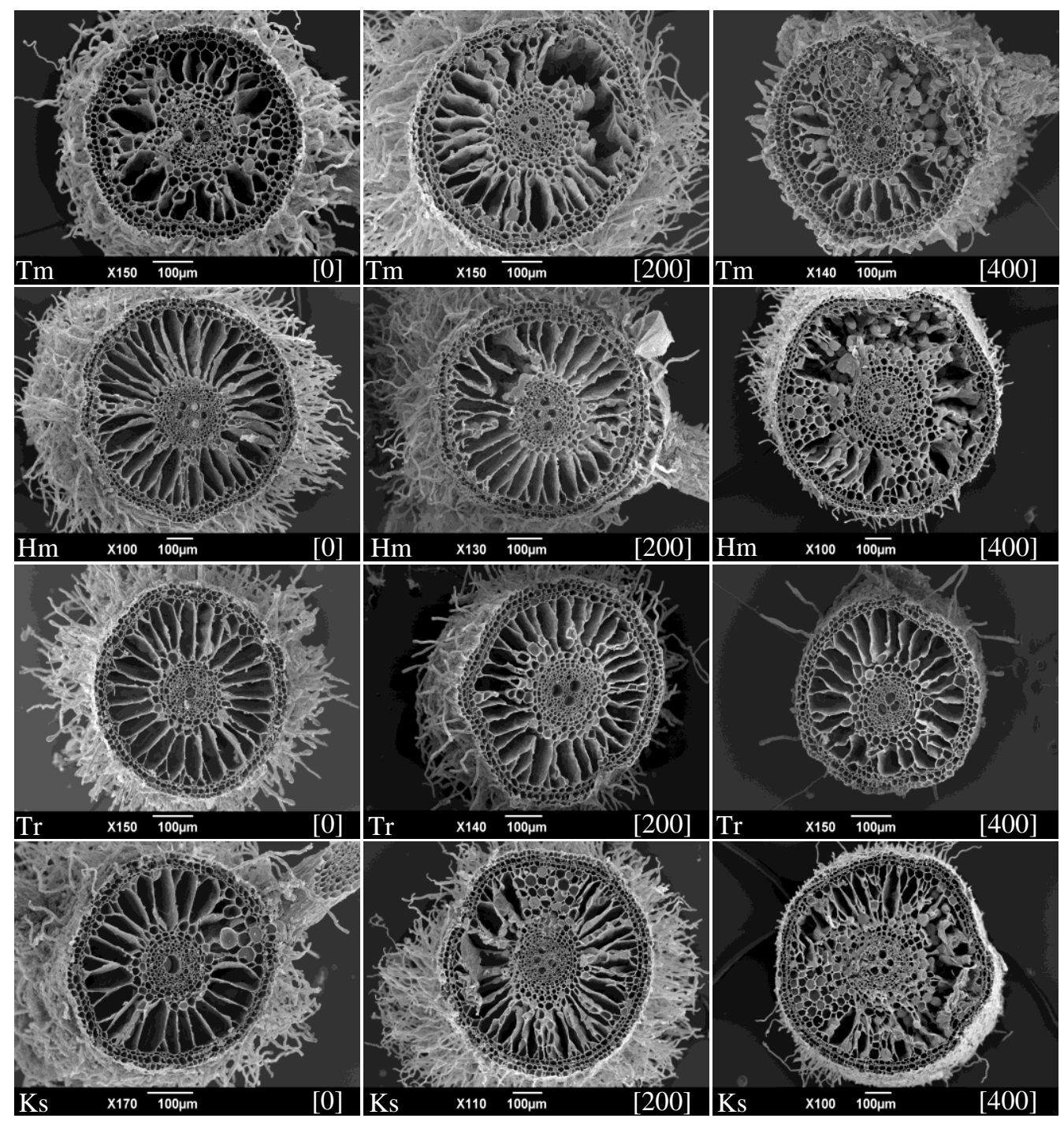

Figura 3. Cortes transversales de raíces de arroz de las variedades 'Temporalero' (Tm), 'Huimanguillo' $(\mathrm{Hm})$, 'Tres Ríos' (Tr) y 'Koshihikari' (Ks), tratadas con tres concentraciones de Al (0, 200 y $400 \mu \mathrm{M}$ de aluminio en forma de $\left.\mathrm{AlCl}_{3}\right)$.

Cuadro 2. Efecto de variedad, concentración de Al y de su interacción, en la concentración nutrimental de $\mathrm{Al}, \mathrm{Ca}, \mathrm{K}, \mathrm{Mg}$ y $\mathrm{P}$ en hojas de arroz.

\begin{tabular}{lccc}
\hline Catión & Fuente de variación & Grados de libertad & Significancia \\
\hline $\mathrm{Al}$ & Variedad & 3 & $* *$ \\
& Concentración de Al & 2 & $* *$ \\
$\mathrm{Ca}$ & Variedad x [Al] & 6 & $* *$ \\
& Variedad & 3 & $* *$ \\
& Concentración de Al & 2 & $* *$ \\
$\mathrm{~K}$ & Variedad x [Al] & 6 & $*$ \\
& Variedad & 3 & $* *$ \\
$\mathrm{Mg}$ & Concentración de Al & 2 & $* *$ \\
& Variedad x [Al] & 6 & $* *$ \\
& Variedad & 3 & $* *$ \\
$\mathrm{P}$ & Concentración de Al & 2 & $* *$ \\
& Variedad x [Al] & 6 & $*$ \\
& Variedad & 3 & $* *$ \\
& Concentración de Al & 2 & $* *$ \\
$\mathrm{HII}$ & Variedad x [Al] & 6 & $*$ \\
\hline
\end{tabular}

[Al] = concentración de Al; $*=$ Significativo a $5 \% ; * *=$ Significativo a $1 \%$. 
Cuadro 3. Concentración de nutrimentos en hojas de cuatro variedades de arroz sometidas a estrés por Al. I: Efecto de la variedad.

\begin{tabular}{lccccc}
\hline \multirow{2}{*}{ Variedad } & \multicolumn{5}{c}{ Concentración de nutrimentos $\left(\mathrm{mg} \mathrm{kg}^{-1}\right)$} \\
\cline { 2 - 6 } & $\mathrm{Al}$ & $\mathrm{Ca}$ & $\mathrm{K}$ & $\mathrm{Mg}$ & $\mathrm{P}$ \\
\hline 'Temporalero' & $0.434 \mathrm{a}^{\dagger}$ & $7.350 \mathrm{a}$ & $18.845 \mathrm{a}$ & $5.326 \mathrm{a}$ & $8.459 \mathrm{a}$ \\
'Huimanguillo' & $0.304 \mathrm{~b}$ & $4.730 \mathrm{~b}$ & $12.019 \mathrm{bc}$ & $3.541 \mathrm{~b}$ & $5.637 \mathrm{c}$ \\
'Tres Ríos' & $0.286 \mathrm{~b}$ & $5.149 \mathrm{~b}$ & $10.097 \mathrm{c}$ & $3.264 \mathrm{~b}$ & $5.006 \mathrm{c}$ \\
'Koshihikari' & $0.251 \mathrm{~b}$ & $4.555 \mathrm{~b}$ & $12.901 \mathrm{~b}$ & $3.402 \mathrm{~b}$ & $6.961 \mathrm{~b}$ \\
\hline
\end{tabular}

${ }^{\dagger}$ Medias con letras distintas en la misma columna indican diferencias estadísticas significativas (Tukey, 0.05).

Cuadro 4. Concentración de nutrimentos en hojas de arroz sometidas a estrés por Al. II: Efecto de la concentración de Al.

\begin{tabular}{lccccc}
\hline Concentración de $\mathrm{Al}$ & \multicolumn{5}{c}{ Concentración de nutrimentos en hoja $\left(\mathrm{mg} \mathrm{kg}^{-1}\right)$} \\
\cline { 2 - 6 } en la solución & $\mathrm{Al}$ & $\mathrm{Ca}$ & $\mathrm{K}$ & $\mathrm{Mg}$ & $\mathrm{P}$ \\
\hline $0 \mu \mathrm{M} \mathrm{Al}$ & $0.114 \mathrm{c}^{\dagger}$ & $6.323 \mathrm{a}$ & $16.311 \mathrm{a}$ & $4.686 \mathrm{a}$ & $7.798 \mathrm{a}$ \\
$200 \mu \mathrm{M} \mathrm{Al}$ & $0.308 \mathrm{~b}$ & $5.501 \mathrm{~b}$ & $13.751 \mathrm{~b}$ & $4.047 \mathrm{~b}$ & $7.086 \mathrm{~b}$ \\
$400 \mu \mathrm{M} \mathrm{Al}$ & $0.533 \mathrm{a}$ & $4.509 \mathrm{~b}$ & $10.332 \mathrm{c}$ & $2.915 \mathrm{c}$ & $4.662 \mathrm{c}$ \\
\hline
\end{tabular}

${ }^{\dagger}$ Medias con letras distintas en la misma columna indican diferencias estadísticas significativas (Tukey, 0.05).

Cuadro 5. Efecto de la interacción variedad x concentración de Al sobre la concentración de nutrimentos en hoja de cuatro variedades de arroz.

\begin{tabular}{|c|c|c|c|c|c|c|}
\hline \multirow[t]{2}{*}{ Variedad } & \multirow{2}{*}{$\begin{array}{c}\text { Concentración } \\
\text { de } \mathrm{Al}\end{array}$} & \multicolumn{5}{|c|}{ Concentración de nutrimentos en hoja $\left(\mathrm{mg} \mathrm{kg}^{-1}\right)$} \\
\hline & & $\mathrm{Al}$ & $\mathrm{Ca}$ & $\mathrm{K}$ & $\mathrm{Mg}$ & $\mathrm{P}$ \\
\hline \multirow[t]{3}{*}{ 'Temporalero' } & 0 & $0.16 \mathrm{fg}^{\dagger}$ & $8.15 \mathrm{a}$ & $19.33 \mathrm{ab}$ & $6.26 \mathrm{a}$ & $10.06 \mathrm{a}$ \\
\hline & 200 & $0.43 \mathrm{~cd}$ & $7.93 \mathrm{a}$ & $18.78 \mathrm{ab}$ & $5.94 \mathrm{ab}$ & $9.12 \mathrm{ab}$ \\
\hline & 400 & $0.71 \mathrm{a}$ & $5.97 \mathrm{bc}$ & $18.42 \mathrm{ab}$ & 3.78 cde & $6.20 \mathrm{def}$ \\
\hline \multirow[t]{3}{*}{ 'Huimanguillo' } & 0 & $0.09 \mathrm{~g}$ & $5.11 \mathrm{bc}$ & $13.67 \mathrm{bcd}$ & $4.36 \mathrm{~cd}$ & $7.07 \mathrm{cde}$ \\
\hline & 200 & $0.21 \mathrm{fg}$ & $4.61 \mathrm{c}$ & $11.32 \mathrm{~cd}$ & $3.46 \mathrm{de}$ & $6.13 \mathrm{def}$ \\
\hline & 400 & $0.61 \mathrm{ab}$ & $4.48 \mathrm{~cd}$ & $11.07 \mathrm{~cd}$ & 2.80 ef & $3.71 \mathrm{~g}$ \\
\hline \multirow[t]{3}{*}{ ‘Tres Ríos’ } & 0 & $0.19 \mathrm{fg}$ & $6.38 \mathrm{~b}$ & $11.78 \mathrm{~cd}$ & $3.17 \mathrm{de}$ & $5.06 \mathrm{fg}$ \\
\hline & 200 & $0.31 \mathrm{de}$ & $4.59 \mathrm{c}$ & $10.62 \mathrm{~cd}$ & $3.35 \mathrm{de}$ & $5.16 \mathrm{efg}$ \\
\hline & 400 & $0.57 \mathrm{bc}$ & $4.48 \mathrm{~cd}$ & $7.89 \mathrm{de}$ & $3.27 \mathrm{de}$ & $4.80 \mathrm{fg}$ \\
\hline \multirow{3}{*}{ 'Koshihikari' } & 0 & $0.13 \mathrm{fg}$ & $5.68 \mathrm{bc}$ & $20.47 \mathrm{a}$ & $4.95 \mathrm{bc}$ & $9.01 \mathrm{abc}$ \\
\hline & 200 & $0.38 \mathrm{de}$ & $4.99 \mathrm{bc}$ & $14.29 \mathrm{bc}$ & $3.45 \mathrm{de}$ & $7.94 \mathrm{bcd}$ \\
\hline & 400 & $0.25 \mathrm{ef}$ & $3.01 \mathrm{~d}$ & $3.95 \mathrm{e}$ & $1.81 \mathrm{f}$ & $3.94 \mathrm{~g}$ \\
\hline
\end{tabular}

${ }^{\dagger}$ Medias con letras distintas en la misma columna indican diferencias estadísticas significativas (Tukey, 0.05).

Al analizar el efecto de las concentraciones de $\mathrm{Al}$ probadas, se observó que a medida que se aumentaba el nivel de $\mathrm{Al}$ en la solución nutritiva, también se incrementaban los niveles de $\mathrm{Al}$ y disminuían las concentraciones de $\mathrm{Ca}, \mathrm{K}, \mathrm{Mg}$ y $\mathrm{P}$ (Cuadro 4). En cuanto a la interacción variedad $\mathrm{x}$ concentración de $\mathrm{Al}$, se observaron diferencias significativas $(\mathrm{P} \leq 0.05)$ sobre la concentración de los nutrimentos estudiados (Cuadro 5). La mayor concentración de $\mathrm{Al}$ se detectó en la variedad 'Temporalero' (0.71 $\mathrm{mg} \mathrm{kg}^{-1}$ ) tratada con $400 \mu \mathrm{M}$ de $\mathrm{Al}$ en solución, en tanto que la menor concentración de este elemento se obtuvo en 'Koshihikari' $\left(0.25 \mathrm{mg} \mathrm{kg}^{-1}\right)$, en mismo tratamiento de Al. Según Ma et al. (2005), 'Koshihikari' es una variedad resistente a $\mathrm{Al}$, lo que puede explicar que haya acumulado menores niveles de $\mathrm{Al}$ en hoja. De las tres variedades mexicanas, 'Tres Ríos' acumuló los menores niveles de $\mathrm{Al}\left(0.57 \mathrm{mg} \mathrm{kg}^{-1}\right)$.

La concentración de los demás nutrimentos disminuyó a medida que se incrementaban los niveles de $\mathrm{Al}$ en la solución nutritiva. 'Koshihikari' registró los menores niveles de $\mathrm{Ca}\left(3.02 \mathrm{mg} \mathrm{kg}^{-1}\right)$ cuando fue tratada con 400 $\mu \mathrm{M}$ Al. De acuerdo con Watanabe y Okada (2005), las aplicaciones de $\mathrm{Ca}$ disminuyen el contenido de $\mathrm{Al}$ en los ápices de raíces, porque este elemento protege a la planta contra la toxicidad por Al. Un comportamiento similar al registrado en Ca se observó para los nutrimentos $\mathrm{K}, \mathrm{Mg}$ y $\mathrm{P}$; es decir, los niveles más bajos de estos elementos se observaron en la variedad 'Koshihikari'. Dado que esta variedad es reportada como resistente a Al y mostró ser la más afectada por los niveles de Al probados, es posible postular que el resto de las variedades, todas ellas mexicanas, muestran mayor tolerancia a este agente limitativo de los suelos ácidos.

De especial interés resulta la variedad 'Tres Ríos', la cual concentró menor cantidad de Al que 'Temporalero' y 'Huimanguillo' (0.57 vs. 0.61 y $0.71 \mathrm{mg} \mathrm{Al} \mathrm{kg}{ }^{-1}$, respectivamente), y no disminuyó sus niveles de $\mathrm{Mg}$ con las aplicaciones de $\mathrm{Al}$, lo cual es importante porque $\mathrm{Mg}$ es un nutrimento que alivia los daños ocasionados por $\mathrm{Al}$ (Yang et al., 2007). Además, 'Tres Ríos' mostró el menor efecto del $\mathrm{Al}$ en la concentración de $\mathrm{P}$, pues las plantas tratadas con $400 \mu \mathrm{M}$ de $\mathrm{Al}$ sólo disminuyeron en 5 $\%$ la concentración de $\mathrm{P}$ respecto a las plantas testigo, en tanto que las plantas de 'Temporalero' disminuyeron su 
contenido de $\mathrm{P}$ en $38 \%$, y las de 'Huimanguillo' lo hicieron en un $55 \%$.

Estos resultados concuerdan con los reportados por Macedo y Jan (2008), quienes evaluaron los efectos de $\mathrm{Al}$ sobre el crecimiento, la absorción y el transporte de macronutrientes $(\mathrm{Ca}, \mathrm{K}, \mathrm{Mg}$ y $\mathrm{P})$ y micronutrientes $(\mathrm{Cu}$, $\mathrm{Fe}, \mathrm{Zn}$ y $\mathrm{Mn}$ ) en arroz, en un $\mathrm{pH}$ de 4.1 , y encontraron que a mayor concentración de $\mathrm{Al}$ ocurre un descenso en la concentración de $\mathrm{Ca}, \mathrm{Mg}$ y $\mathrm{Mn}$, así como un incremento en $\mathrm{Fe}, \mathrm{Cu}$ y $\mathrm{Zn}$. Ramachandran et al. (2004) observaron que con concentraciones de 160 y $320 \mu \mathrm{g} \mathrm{m}^{-1}$ de $\mathrm{Al}$ se redujeron las concentraciones de $\mathrm{P}, \mathrm{Ca}, \mathrm{Zn}$ y $\mathrm{Mn}$, lo cual coincide con lo aquí encontrado.

Aunque el arroz es considerado un cultivo tolerante a Al (Ishikawa et al., 2000; Kikui et al., 2005), su nivel de tolerancia difiere entre variedades (Jan y Pettersson, 1993; Matsumoto, 2000), y su mecanismo de tolerancia sigue siendo investigado. Según Okada et al. (2003) el mayor rendimiento de variedades sensibles a $\mathrm{Al}$ correlacionó positivamente con el $\mathrm{Ca}$ intercambiable en suelos altamente degradados con baja capacidad de intercambio catiónico, lo que sugiere que el $\mathrm{Ca}$ tiene un papel importante en suelos ácidos y en la tolerancia del arroz a Al, lo cual es también reportado por Watanabe y Okada (2005). Por otra parte, la deficiencia de $\mathrm{Mg}$ inducida por $\mathrm{Al}$ se ha asociado con inhibición de la absorción de Mg (Huang et al., 2009) por el bloqueo proteínas transportadoras de membrana (Matsumoto, 2000). Yang et al. (2007) demostraron que el Mg mitiga los síntomas de toxicidad de Al en Vigna umbellata, al ayudar a mantener niveles considerables de $\mathrm{Ca}$ y $\mathrm{Mg}$ en los ápices de raíces.

En años recientes se han dado importantes avances en el estudio de las bases moleculares de la tolerancia a Al. Ma et al. (2005) aislaron mutantes sensibles a $\mathrm{Al}$ a partir de líneas $\mathrm{M}(3)$ derivadas de la variedad 'Koshihikari' resistente a $\mathrm{Al}$, irradiadas con rayos gamma. Larsen et al. (2005) descubrieron que la proteína ALS3, un transportador tipo "cassette" de unión a ATP (o transportador $\mathrm{ABC}$ ), está involucrada en el transporte de $\mathrm{Al}$ de la raíz hacia tejidos menos sensibles a Al. Zhang et al. (2007) reportaron que a nivel transcripcional, la desintegración del citoesqueleto puede estar asociada con la toxicidad por $\mathrm{Al}$, en tanto que el transporte de iones y el metabolismo de $\mathrm{S}$ pueden tener importantes funciones en la tolerancia a Al en arroz. Según Rounds y Larsen (2008), la inhibición del crecimiento de la raíz expuesta a $\mathrm{Al}$ surge del daño al DNA acoplado con un bloqueo del ciclo celular controlado por la proteína AtATR. Huang et al. (2009) reportaron que los genes STARI (sensitive to Al rhizotoxicity1) y STAR2, son responsables de la tolerancia a $\mathrm{Al}$ en arroz.
STARI codifica un dominio de unión a nucleótido, en tanto que STAR2 codifica un dominio transmembrana de un transportador tipo ABC. Las proteínas STAR1 y STAR2 forman un complejo que funciona como transportador $\mathrm{ABC}$, el cual se requiere para desintoxicar al arroz del Al. El transportador tipo ABC transporta UDPglucosa, molécula que puede ser usada para modificar la pared celular.

En futuras investigaciones se dará prioridad al estudio de los mecanismos moleculares involucrados en las respuestas diferenciales al aluminio de las variedades de arroz aquí analizadas, con énfasis en genes que pudieran estar codificando proteínas que controlen la expresión de genes como STAR1, STAR2 y $A L S 3$ involucradas en la tolerancia a $\mathrm{Al}$; algunas de estas proteínas podrían ser factores de transcripción, de los cuales existen más de 2500 en el proteoma del arroz (Riaño-Pachón et al., 2008). De especial importancia para México resultan estos estudios, pues tres de las cuatro variedades analizadas son mexicanas y, una de ellas, la variedad 'Tres Ríos', muestra mayor tolerancia que la variedad 'Koshihikari' considerada tolerante a Al.

\section{CONCLUSIONES}

El incremento de la concentración de Al en la solución nutritiva afecta variables fisiológicas y nutrimentales en arroz, como altura de planta, estructura de la raíz y absorción de nutrimentos. El efecto del Al depende de su concentración en la solución nutritiva y de la variedad. La menor altura de planta fue observada en la var. 'Koshihikari' tratada con $400 \mu \mathrm{M}$ de $\mathrm{Al}$ en la solución, en tanto que la var. 'Temporalero' mostró menor afectación de esta variable en el mismo tratamiento $(400 \mu \mathrm{M})$. Con excepción de 'Tres Ríos', las demás variedades presentaron daños a nivel tejido de raíz. Ambos factores y sus interacciones afectaron significativamente la concentración de $\mathrm{Al}, \mathrm{Ca}, \mathrm{K}, \mathrm{Mg}$ y $\mathrm{P}$ en la hoja. De las variedades mexicanas evaluadas, 'Tres Ríos' concentró menor cantidad de Al, no varió sus niveles de $\mathrm{Mg}$ en hoja por efecto de $\mathrm{Al}$, su concentración foliar de $\mathrm{P}$ fue menos afectada, $\mathrm{y}$ no presentó daños estructurales en la raíz, por lo que se concluye que es una variedad con amplias posibilidades para ser cultivada en suelos ácidos.

\section{AGRADECIMIENTOS}

Al Consejo Nacional de Ciencia y Tecnología (CONACYT) por los apoyos otorgados a FCGM en el esquema de repatriación y por la beca para estudios de maestría en ciencias conferida a TMG. A las Líneas Prioritarias de Investigación 5 "Biotecnología microbiana, vegetal y animal" y 16 "Innovación tecnológica" del 
Colegio de Postgraduados, por las facilidades brindadas.

\section{BIBLIOGRAFÍA}

Aimi H, M Uetake, K Shimizu (2009) Effective combinations of functional groups in chemically modified kraft lignins for reduction of aluminum toxicity. J. Wood Sci. 55:220-224.

Alcántar G G, M Sandoval V (1999) Manual de Análisis Químico de Tejido Vegetal. Sociedad Mexicana de la Ciencia del Suelo A.C. Publicación especial No. 10. Chapingo México. 156 p.

Blancaflor E B, D L Jones, S Gilroy (1998) Alterations in the cytoskeleton accompany aluminum-induced growth inhibition and morphological changes in primary roots of maize. Plant Physiol. 118:159-172.

Du Y M, J Tian, H Liao, C J Bai, XL Yan, G D Liu (2009) Aluminium tolerance and high phosphorus efficiency helps Stylosanthes better adapt to low-P acid soils. Ann. Bot. 103:1239-1247.

Echeverría-Rubio E (2003) Desarrollan un maíz resistente al aluminio. Investigación y Desarrollo 189:7-8.

Hirano Y, N Hijii (1998) Effects of low $\mathrm{pH}$ and aluminum on root morphology of Japanese red cedar saplings. Environ. Poll. 101:339-347.

Huang C F, N Yamaji, N Mitani, M Yano, Y Nagamura, J F Ma (2009) A bacterial-type $A B C$ transporter is involved in Aluminum tolerance in rice. Plant Cell 21:655-667.

Ishikawa S, T Wagatsuma, R Sasaki, P Ofei-Manu (2000) Comparison of the amount of citric and malic acids in $\mathrm{Al}$ media of seven plant species and two cultivars each in five plant species. Soil Sci. Plant Nutr. 46:751-758.

Jan F (2005) Aluminium effects on growth, nutrient net uptake and transport in 3 rice (Oryza sativa) cultivars with different sensitivity to aluminium. Physiol. Plant. 83:441-448.

Jan F, S Pettersson (1993) Effect of low aluminum levels on growth and nutrient relations in three rice cultivars with different tolerances to aluminum. J. Plant Nutr. 16:359-372.

Kikui S, T Sasaki, M Maekawa, A Miyao, H Hirochika, H Matsumoto, Y Yamamoto (2005) Physiological and genetic analyses of aluminium tolerance in rice, focusing on root growth during germination. J. Inorg. Biochem. 99:1837-1844.

Kochian L V, O A Hoekenga, M A Piñeros (2004) How do crop plants tolerate acid soil? Mechanisms of aluminum tolerance and phosphorous efficiency. Annu. Rev. Plant Biol. 55:459493.

Larsen P B, M J B Geisler, C A Jones, K M Williams, J D Cancel (2005) $A L S 3$ encodes a phloem-localized $\mathrm{ABC}$ transporter-like protein that is required for aluminum tolerance in Arabidopsis. Plant J. 41:353-363.

Lenoble M E, G Blevins, E Sharp, G Cumbie (1996) Prevention of aluminium toxicity with supplemental boron. I. Maintenance of root elongation and cellular structure. Plant Cell Environ. 19:1132-1142

Liu K, S Luan (2001) Internal aluminum block of plant inward $\mathrm{K}^{+}$ Channels. Plant Cell 13:1453-1466.

Ma J F, R Shen, Z Zhao, M Wissuwa, Y Takeuchi, T Ebitani (2002) Response of rice to Al stress and identification of quantitative trait loci for $\mathrm{Al}$ tolerance. Plant Cell Physiol. 43:652-659.

Ma J F, S Nagao, C F Huang, M Nishimura (2005) Isolation and characterization of a rice mutant hypersensitive to Al. Plant
Cell Physiol. 46:1054-1061.

Macedo C E C, V V S Jan (2008) Effect of aluminum stress on mineral nutrition in rice cultivars differing in aluminum sensitivity. Rev. Bras. Eng. Agric. Amb. 12:363-369.

Malkanthi D R R, M Moritsugu, K Yokoyama (1995) Effects of low $\mathrm{pH}$ and $\mathrm{Al}$ on absorption and translocation of some essentia nutrients in excised barley roots. Soil Sci. Plant Nutr. 41:253 262.

Matsumoto H (2000) Cell biology of aluminum toxicity and tolerance in higher plants. Int. Rev. Cytol. 200:1-46.

Matsumoto H, Y Senoo, M Kasai, M Maeshima (1996) Response of the plant root to aluminum stress: Analysis of the inhibition of the root elongation and changes in membrane function. J. Plant Res. 109:99-105.

Okada K, A J Fischer, F A Perez Salasar, Y Cañon Romero (2003) Difference in the retention of $\mathrm{Ca}$ and $\mathrm{Al}$ as possible mechanisms of $\mathrm{Al}$ resistance in upland rice. Soil Sci. Plant Nutr. 49:889-895.

Poschenrieder C, B Gunsé, I Corrales, J Barceló (2008) A glance into aluminum toxicity and resistance in plants. Sci. Total Environ. 400:356-368.

Ramachandran V, D U Patel, S C Kumar (2004) Influence of aluminium on mineral nutrients of rice seedlings grown in solution culture. Indian J. Plant Physiol. 9:348-353.

Rengel Z (1992) Role of calcium in aluminium toxicity. New Phytol. 121:499-513

Riaño-Pachón D M, L G Guedes-Correa, R Trejos-Espinosa, B Mueller-Roeber (2008) Green Transcription Factors: A Chlamydomonas overview. Genetics 179:31-39.

Rounds M A, P B Larsen (2008) Aluminum-dependent root-growth inhibition in Arabidopsis results from AtATR-regulated cellcycle arrest. Curr. Biol. 18:1495-1500.

SAS (1990) SAS/STAT User's guide. Ver. 6. SAS Institute, Inc. Cary, NC $1686 \mathrm{p}$.

SIAP (2009) Servicio de información agropecuaria y pesquera. México. Disponible en: http://www.siap.gob.mx/ (noviembre de 2009).

Steiner A A, H van Winden (1970) Recipe for ferric salts of ethylenediaminetetraacetic acid. Plant Physiol. 46:862-863.

Sun Q B, R F Shen, X Q Zhao, R F Chen, X Y Dong (2008) Phosphorus enhances Al resistance in Al-resistant Lespedeza bicolor but not in Al-sensitive $L$. cuneata under relatively high Al stress. Ann. Bot. 102:795-804.

Tavitas-Fuentes L, Hernández-Aragón L (2000) Catálogo de Variedades de Arroz que Actualmente se Cultivan en México. INIFAP México. Publicación Especial no. 24. 56 p.

Watanabe T, K Okada (2005) Interactive effects of $\mathrm{Al}$, Ca and other cations on root elongation of rice cultivars under low $\mathrm{pH}$. Ann. Bot. 95:379-385.

Yamamoto Y, S Rikiishi, Y C Chang, K Ono, M Kasai, H Matsumoto (1994) Quantitative estimation of Aluminum toxicity in cultured tobacco cells: correlation between Aluminum uptake and growth inhibition. Cell Physiol. 35:575583.

Yang J L, J F You, Y Y Li, P Wu, S J Zheng (2007) Magnesium enhances aluminum-induced citrate secretion in rice bean roots (Vigna umbellata) by restoring plasma membrane $\mathrm{H}^{+}$-ATPase activity. Plant Cell Physiol. 48:66-73.

Zhang J, Z He, H Tian, G Zhu, X Peng (2007) Identification of aluminium-responsive genes in rice cultivars with different aluminium sensitivities. J. Exp. Bot. 58: 2269-2278. 